\title{
Validation of assays for inflammatory mediators in sputum
}

\author{
R.A. Stockley, D.L. Bayley
}

Validation of assays for inflammatory mediators in sputum. R.A. Stockley, D.L. Bayley. (C) ERS Journals Ltd 2000.

ABSTRACT: Commercially available immune assays are being used with increasing frequency in the study of lung inflammation. However, their performance in complex biological fluids is rarely assessed. The authors wished to assess their reliability to determine whether the results obtained in sputum samples can be easily interpreted.

The reproducibility of several such assays was therefore determined together with their ability to recover known amounts of pure reagent. Sputum sol phase was obtained from several patients with chronic lung disease and used together with the reagents in a series of "spiking" and dilutional experiments.

Results confirmed that the enzyme assay for myeloperoxidase and the immune assays for interleukin-8, leukotriene $B_{4}$ and secretory leukoproteinase inhibitor were all reproducible (intra-assay coefficient of variation 3.8-7.7\%). Furthermore, each of these assays gave $>\mathbf{8 5} \%$ recovery of a "spike" with pure reagent. However, the immune assay for myeloperoxidase (although reproducible) gave poor recovery and was dependent on the degree of sample dilution and elastase content.

These studies confirm that the reliabilities of fluid phase measurements should be assessed before being widely applied to complex biological samples. Eur Respir J 2000; 15: 778-781.
Dept of Medicine, Queen Elizabeth Hospital, Birmingham, UK.

Correspondence: R.A. Stockley, Dept of Medicine, Queen Elizabeth Hospital, Edgbaston, Birmingham, B15 2TH, UK. Fax: 441216978257

Keywords: Bronchial secretions cytokines

inflammation

Received: October 221998

Accepted after revision November 251999

This research was supported by a noncommercial grant from Bayer Plc (West Haven, CT, USA) as part of the Antitrypsin Deficiency Assessment and Programme for Treatment (ADAPT) project.
In recent years, there has been increasing interest in the role of lung inflammation in the development and progression of a variety of lung diseases as diverse as pulmonary fibrosis [1], cystic fibrosis [2], idiopathic bronchiectasis [3], chronic obstructive pulmonary disease (COPD) [4] and asthma [5]. These studies have been facilitated by the widespread availability of commercial assays to measure the inflammatory mediators of interest. These assays have been validated and their performance is described within the company literature. However, the validation and reproducibility has usually been determined in vitro. On the other hand, the measurements are usually made in biological fluids and confirmation of their reproducibility within these fluids is rarely quoted. This is of major importance, particularly since protein recovery may not be complete and the presence of inflammation will be associated with a variety of proteolytic enzymes which may in themselves interfere with the assay accuracy.

It has always been the authors' practice to validate new assays for clinical research. This has involved the spiking of biological fluids with pure mediator and subsequent assessment of the stability of the measurement and the proportion of the recovery. The authors report here some validation experiments using expectorated sputum sol phase for the assessment of myeloperoxidase (by enzymatic and immune assay), the chemoattractants interleukin (IL)- 8 and leukotriene $\mathrm{B}_{4}\left(\mathrm{LTB}_{4}\right)$ and the proteinase inhibitor secretory leukoprotease inhibitor (SLPI) (all by immune assay). In addition, the authors have studied the effect of neutrophil elastase on assay performance in the absence of a biological fluid. The assays selected were already in use within the authors' laboratory or were easily available at the time of the study. Since the completion of this study several companies have brought similar assays to market. However, the authors are yet to complete any comparison with the previously studied assays.

\section{Methods}

Sputum sol phase was obtained by ultracentrifugation of spontaneously expectorated samples as described previously [3]. A pool of mucoid sputum sol phase was obtained from a group of eight patients with stable COPD. In addition, a pool of mucopurulent sol phase was obtained from a group of eight patients with bronchiectasis.

\section{Elastase activity assay}

Elastase activity was determined for each sputum sol phase pool by the use of the chromogenic substrate $N$ succinyl-Ala-Ala-Ala p-nitroanilide, using the method described by McGiLLIVRAY et al [6].

\section{Assay validation}

All assays were performed in a similar way. Firstly, a standard curve was obtained using a pure preparation of the mediator provided by the manufacturer. Following this, a sample of the mucoid and mucopurulent sputum sol phase pool was assayed and the result compared to the standard curve. The value for the mediator was then 
obtained by interpolation. Single samples were assayed on 4-6 occasions to obtain the intra-assay coefficient of variation. At this point, a known quantity of the pure mediator was added to the sample and it was reassayed. Again, a result was obtained and compared to the standard curve and the result for the new value obtained by interpolation. This second value was subtracted from the predicted value to obtain the proportion "recovered".

The effect of sample dilution was compared by assaying the same sputum sol phase at different dilutions from 1 in 20 to 1 in 80 for the mucoid sputum and 1 in 100 to 1 in 400 for the mucopurulent sol phase (each dilution measured four times). All dilutions provided results within the working range of the relevant assay. The protein value was obtained as above and corrected for the dilution to determine whether the dilution alone had an effect upon the mediator value obtained.

Finally, a known amount of the mediator was mixed with increasing concentrations of active neutrophil elastase from $10^{-9}-10^{-6} \mathrm{M}$ to span the range of values found previously in lung secretions. The mixture was again assayed using the standard curves to determine whether there was any change in the value obtained.

\section{Myeloperoxidase}

Myeloperoxidase concentration was measured by two methods. The first utilized a commercial immune assay obtained from R \& D Systems Europe Ltd, (Abingdon, Oxfordshire, UK) (expressed as ng. $\mathrm{mL}^{-1}$ ). The second assay was based upon the activity of myeloperoxidase (MPO) as measured by chromogenic substrate assay. In brief, $10 \mathrm{~mL}$ of the standard or sample were added to the wells of a microtitre plate. One hundred and fifty microlitres of 1 $\mathrm{mg} \cdot \mathrm{mL}^{-1}$. (weight $(\mathrm{w}) /$ volume $(V)$ ) o-dianisidine dihydrochloride, $0.01 \%$ (V/V) $30 \% \mathrm{H}_{2} \mathrm{O}_{2}$ in $50 \mathrm{mM} \mathrm{K}_{3} \mathrm{HPO}_{4}$ and $0.5 \%(\mathrm{w} / V)$ hexadecyl trimethyl ammonium bromide PH6 (Sigma Aldrich Co. Ltd., Poole, Dorset, UK) was then added to each well. The plate was incubated for $15 \mathrm{~min}$ at $25 \mathrm{C}$, the absorbance was measured at $450 \mathrm{~nm}$ measured and the MP0 activity obtained by interpolation from the standard curve (expressed as units of activity per millilitre).

The chemoattractants IL-8 (D8050; Quantiline, Abingdon, UK), LTB4 (Amersham International plc., Little Chalfont, Buckinghamshire, UK) and SLPI (R \& D Systems Europe Ltd.) were all measured using commercially available kits according to the manufacturers' instructions. Data from the IL-8, $\mathrm{LTB}_{4}$ and SLPI assays expressed as nanograms per millilitre.

\section{Results}

The mucoid sputum sol phase pool contained no free elastase activity detected with the current substrate (see Methods section). However the elastase activity of the mucopurulent sol phase pool was $2.13 \mu \mathrm{M}$.

Good standard curves were obtained for all assays and the correlation coefficient (r) for the standard curves varied between 0.990-0.998. The intraassay coefficient of variation showed some differences across the range of the assays, varying from $12 \%$ at very low levels to $<3 \%$ at high levels.

In general, the recovery of the mediator from samples spiked with known amounts was good. Figures 1-3 show the relationship of the assay result to the mediator con- centration or activity. Results generally indicate that "spiking" of the samples led to results that were very close to the standard curve, indicating that recovery was virtually complete (generally $>85 \%$ ). However, "spiking" of samples using the MPO immune assay resulted in values clearly displaced from the standard curve (fig. 4). When the actual values obtained by interpolation from the standard curve were assessed, the recovery ranged 28.5$69.8 \%$ of the MPO added to the sample. The results demonstrated that best recovery was obtained in mucopurulent sputum which had been diluted 200 times prior to addition of the MPO "spike" and least recovery occurred in samples of mucoid sputum diluted only 40 -fold. In view of this, the authors compared the results for the mucoid samples and mucopurulent samples diluted sequentially. Results indicated that mucoid samples diluted 1 in 20 gave values of $391.0 \mathrm{ng} \cdot \mathrm{mL}^{-1}(\mathrm{SD} \pm 9.4 ; \mathrm{n}=4)$. When diluted 40-fold, the relevant value was 712.7 ( $\mathrm{SD} \pm 8.6$; $\mathrm{n}=4$ ) and when diluted 80 -fold, the value was 1184.7 $(\mathrm{SD} \pm 81.6 ; \mathrm{n}=4)$.

On the other hand, studies with mucopurulent sputum ( $\mathrm{n}=4$, all dilutions) showed that a 1 in 100 dilution provided a result of $4556.5(\mathrm{SD} \pm 59.5): 1$ in 200 dilution 8659.4 $(\mathrm{SD} \pm 613.6)$ and 1 in 400 dilution 13494.4 ( $\mathrm{SD} \pm 250.7)$. Dilutional studies using all other assays resulted in the appropriate and expected value.

Finally, the addition of increasing amounts of elastase to pure mediator had no effect on assays of MPO activity, IL-8 or $\mathrm{LTB}_{4}$ (respectively, $103.4 \%, 96.4 \%$ and $106.2 \%$ of control value at $10^{-6} \mathrm{M}$ elastase). Low concentrations of elastase $\left(\leq 10^{-9} \mathrm{M}\right)$ had no significant effect on the measurement of SLPI $\left(96.2 \%\right.$ of control value at $10^{-9} \mathrm{M}$ elastase). However, with increasing concentrations of elastase, the value of the standard sample dropped dramatically leading to little measurable protein in the presence of $10^{-6} \mathrm{M}$ elastase $\left(4.3 \%\right.$ of control value at $10^{-6} \mathrm{M}$ elastase).

Similar studies using the MPO enzyme linked immunosorbent assay (ELISA) indicated that even concentrations as low as $10^{-9} \mathrm{M}$ (below the lower limit of detection for the assay reported here) resulted in a significant loss of

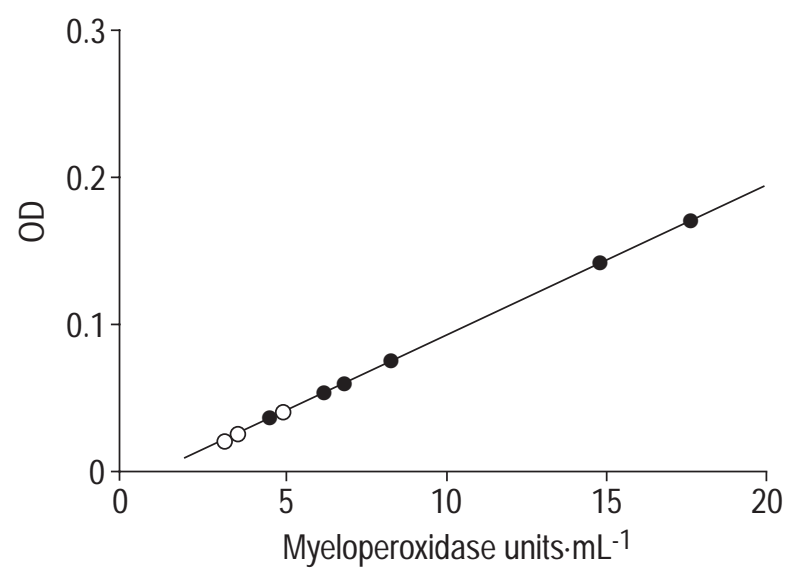

Fig. 1. - The enzyme assay for myeloperoxidase (MPO) is shown together with the regression line for the laboratory standards. The vertical axis is the optical density (OD) of the reaction (see Methods section) and the horizontal axis is the enzyme activity. $\bigcirc$ : biological samples; : the OD obtained by adding known amounts of pure enzyme; - : standards. The intra-assay coefficient of variation for this assay was $3.8 \%(n=6)$. 

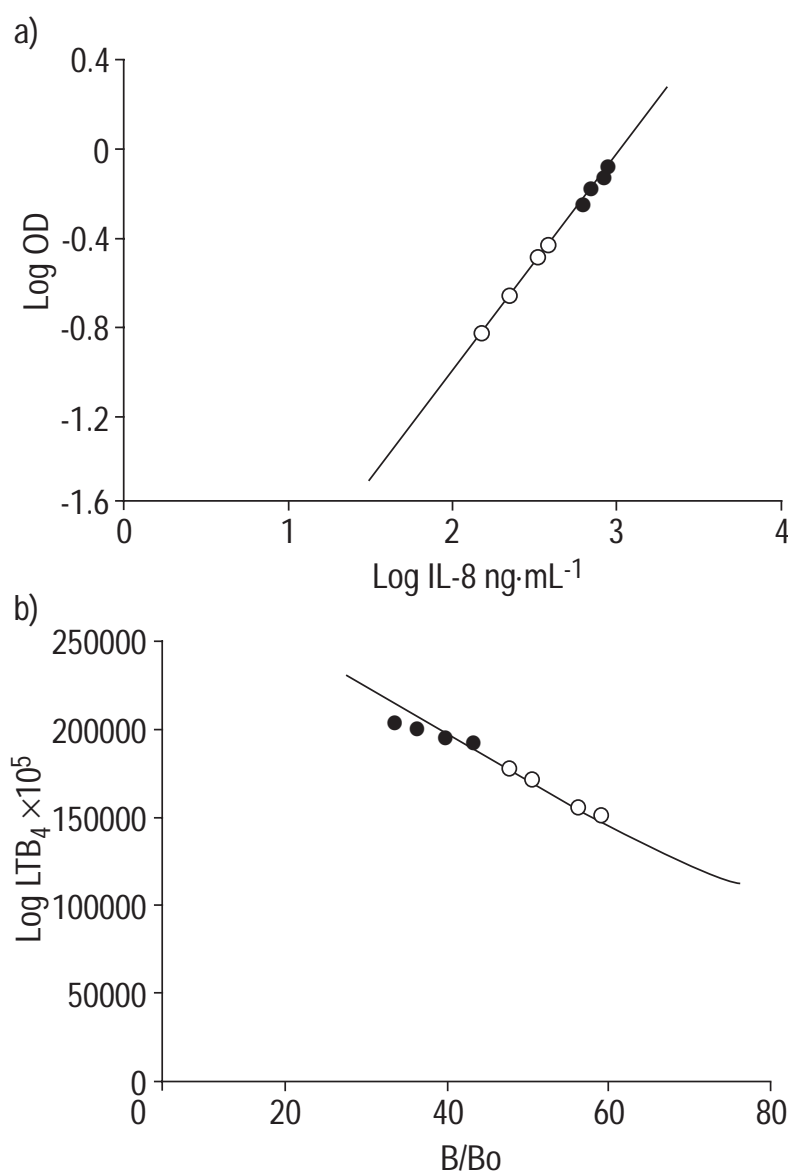

Fig. 2. - Standard curves for the chemoattractants are shown together with results for biological fluids $(O)$. 9 : the relevant reading for samples "spiked" with known amounts of chemoattractant a) interleukin (IL)-8; b) leukotriene (LT)B $\mathrm{B}_{4}$; : standards. B/Bo: (standard or sample optical density (OD) -nonspecific binding OD)/(blank OD nonspecific binding OD). The intra-assay coefficient of variation for these assays were:a) $7.7 \%(n=6)$; and b) $6.3 \%(n=4)$.

MPO (81.6\% recovery). This fell further with increasing concentrations of elastase, resulting in $56.7 \%$ recovery in the presence of $10^{-6} \mathrm{M}$ elastase.

\section{Discussion}

The measurement of mediators in bronchial fluids is providing a major insight into the understanding of the inflammatory process and tissue destruction in many acute and chronic lung disease. However, the role of these mediators, the influence of the presence of disease and response to treatment are clearly dependant on the accuracy of the assays used. The current paper indicates that several of the assays commercially available are both reproducible within assay and appear to measure the protein within the secretion accurately. In most of these assays, the standard curve is linear or can be linearized by the use of logarithmic transformation of data. However, the MPO assay (ELISA) is more complex with a curvilinear relationship between the concentration of the standard and the optical density. In addition, sample dilution and "spiking" samples with known amounts of protein result in variable values and poor recovery. Furthermore, the value obtained is influenced by the degree of sputum sol phase dilution

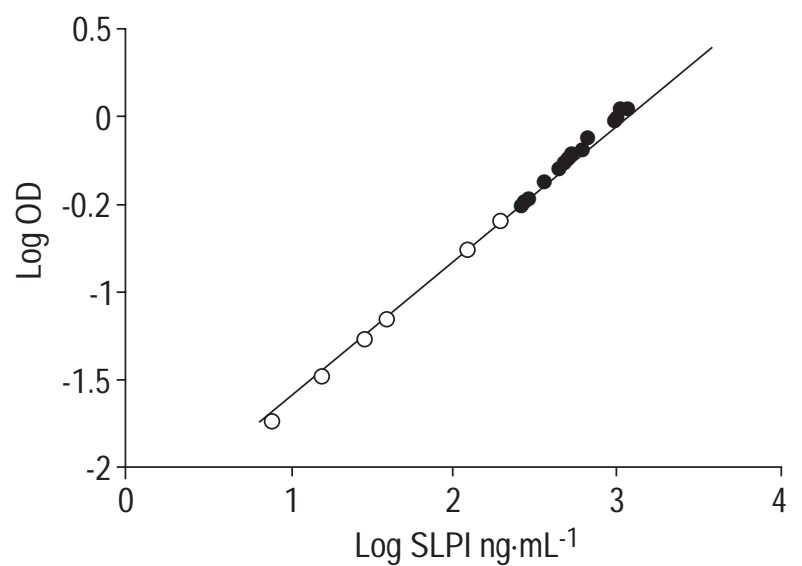

Fig. 3. - Data for biological fluids before $(\bigcirc)$ and after "spiking" with secretary leukoprotease inhibitor (SLPI). The line represents the standard curve. The intra-assay coefficient of variation was $6.4 \%(n=6)$. OD: optical density; _ : standards.

which could reflect interference with the assay or the presence of enzymes such as neutrophil elastase that could potentially result in antibody cleavage, thereby resulting in a low assay signal. Indeed studies with pure neutrophil elastase indicate that increasing concentrations of the enzyme do lead to a reduction in the measurement of known amounts of MPO.

It could be argued that significant dilution of the sample would reduce the elastase concentration sufficiently to remove any interference with the assay performance as with the SLPI assay (see below). An alternative strategy could be to inhibit the elastase activity using an appropriate specific inhibitor such as a chloromethylketone [7]. Nevertheless, even in mucoid sputum, where elastase activity is not a problem, sample dilution led to variable results and variable recovery. Whereas this may not be a problem in samples where the concentration of MPO is known, it clearly presents a significant problem in samples where it is unknown or may be changing with clinical status.

The only other assay described here that was influenced by the presence of elastase activity was that for SLPI.

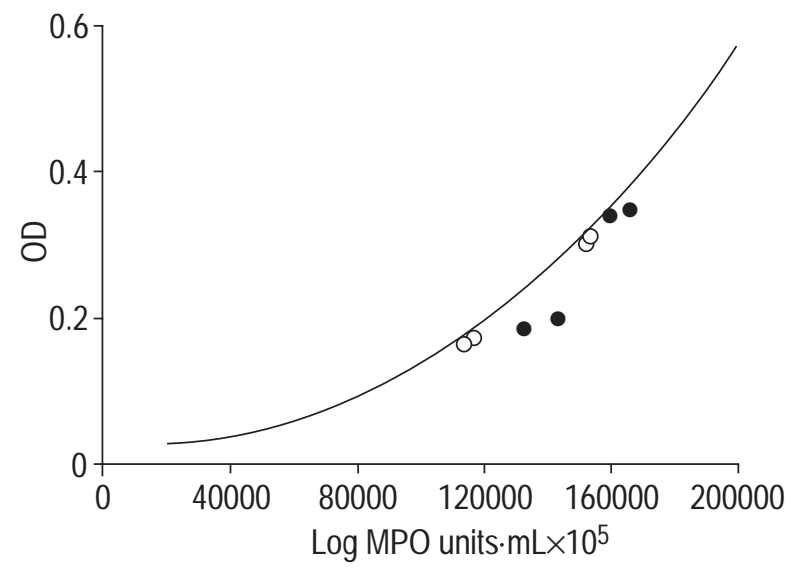

Fig. 4. - Results for the immune assay for myeloperoxidase (MPO) are shown with the standard curve. Note the results for samples "spiked" with pure MPO (O) are displaced to the right of the standard curve giving low optical density (OD) and hence underestimating the amount of enzyme added. The intra-assay coefficient of variation for a single sample was $3.8 \%(\mathrm{n}=6)$. $\bigcirc$ : samples; __ : standards. 
However, the concentration of this protein in biological fluids is high and sample assessment requires significant dilution (usually 1 in 50,000 or more) prior to the measurement of the sample. At these dilutions, even samples containing a large amount of free elastase activity would lead to concentrations $<10 \mathrm{~nm}$ in the final assay mixture (which does not influence its measurement, indicating that it is suitable for assays in bronchial secretions).

If enzyme activity of the sample accounts for low signal return it could be argued that all immune assays would be equally affected. However, neutrophil elastase activity is dependant on both salt concentration and $\mathrm{pH}$ for its activity. In addition all manufacturers' assay buffers contain unidentified proteins as blocking agents. All these factors will influence the characteristics of the immune assay and their susceptibility to signal recovery. Unfortunately it is rarely feasible to obtain the relevant information to enable the performance of an individual assay to be predetermined. Thus validation using the appropriate biological fluids remains critical.

In summary, the data show that dilution perhaps due to the presence of enzyme activity can have a significant effect on some commercially available assays for important mediators in lung disease. It therefore remains critical that validation is carried out prior to using these assays for the measurement of mediators in any biological fluid.

Acknowledgements. The authors thank C. Seymour for typing the manuscript.

\section{References}

1. Yamanouchi H, Fujitu J, Hojo S, et al. Neutrophil elastase: alpha-1-proteinase inhibitor complex in serum and bronchoalveolar lavage fluid in patients with pulmonary fibrosis. Eur Respir J 1998; 11: 120-125.

2. Koller DY, Nething I, Otto J, Urbanek R, Eichler I. Cytokine concentrations in sputum from patients with cystic fibrosis and their relation to eosinophil activity. $\mathrm{Am}$ J Respir Crit Care Med 1997; 155: 1050-1054.

3. Mikami M, Llewellyn-Jones CG, Bayley D, Hill SL, Stockley RA. The chemotactic activity of sputum from patients with bronchiectasis. Am J Resp Crit Care Med 1998; 157: 723-728.

4. Keatings VM, Collins PD, Scott DM, Barnes PJ. Differences in interleukin- 8 and tumour necrosis factora in induced sputum from patients with chronic obstructive pulmonary disease or asthma. Am J Respir Crit Care Med 1996; 153: 530-534.

5. Keatings VM, O'Connor BJ, Wright LG, Hutson DP, Corrigan CJ, Barnes PJ. Late response to allergen is associated with increased concentrations of tumour necrosis factor-alpha and IL-5 in induced sputum. $J$ Allergy Clin Immunol 1997; 99: 693-698.

6. McGillivray DH, Burnett D, Afford SC, Stockley RA. An evaluation of four methods for the measurement of elastase activity. Clin Chim Acta 1981; 111: 289-294.

7. Burnett D, Afford SC, Campbell EJ, Rios-Mollineda RA, Buttle DJ, Stockley RA. Evidence for lipid-associated serine proteases and metalloproteases in human bronchoalveolar lavage fluid. Clin Sci 1989; 77: 35-41. 